\title{
Tolerance of Some Wheat Varieties to Boron Toxicity
}

\author{
S.A. Ghaffari NeJaD ${ }^{1}$, G.R. Savaghebi ${ }^{1}$, M. Farahbakhsh ${ }^{1 *}$, R. MaAli Amiri ${ }^{1}$ and H. Rezaei ${ }^{2}$ \\ ${ }^{1}$ University College of Agriculture and Natural Resources, University of Tehran, 31587-77871, Karaj, Iran \\ ${ }^{2}$ Soil and Water Research Institute, 31587-77871, Karaj, Iran
}

(Received 12 June 2014; Accepted 31 October 2014;

Communicated by A. Anioł)

\begin{abstract}
Boron (B) toxicity is an important problem in low rainfall and highly alkaline soils of central and southern part of Iran. We evaluated B toxicity tolerance of 10 Iranian wheat varieties in a greenhouse experiment. Experimental design was factorial Completely Randomized Design (CRD) with 10 wheat varieties $\times$ six B levels $(0,2.5,5,10,20$ and 40 $\mathrm{mg} \mathrm{B} \mathrm{kg}{ }^{-1}$ soil) in three replications. The results showed a great range of tolerance among wheat varieties. Arg was the most tolerant one and Chamran showed the least tolerance. It seems that different mechanisms involved in B toxicity tolerance, namely exclusion of B from root, redistribution of $\mathrm{B}$ within leaves and integration of these two mechanisms. Significant negative linear correlation observed between shoot B concentration and shoot dry weight $(\mathrm{r}=0.85, p<0.01)$ and positive linear correlation between shoot $\mathrm{B}$ concentration and shoot dry weight reduction percentage $(\mathrm{r}=0.82, p<0.01)$.
\end{abstract}

Keywords: boron toxicity, wheat varieties, boron tolerance

\section{Introduction}

Boron (B) is one of the essential micronutrients for plant growth, widely distributed in lithosphere and hydrosphere. $\mathrm{B}$ is known to be unusual micronutrient in that the range between toxicity and deficiency is narrow and at physiological and common soil pHs exists as un dissociated boric acid (Camacho-Cristóbal et al. 2008; Yau and Ryan 2008; Herrera-Rodríguez et al. 2010; Reid 2010).

Both B deficiency and toxicity can suppress plant growth. B deficiency can be easily corrected but $\mathrm{B}$ toxicity is a major micronutrient disorder affecting yield of many crops especially cereals around the world (Kalayci et al. 1997; Savić et al. 2013). Excessive B in soil and irrigation water causes B toxicity in crops and more over it is a common nutritional problem in low rain fall and dry areas especially in alkaline soils of Australia, North Africa and West Asia (Nable et al. 1997; Camacho-Cristóbal et al. 2008). B toxicity is probably first reported in the united states but its importance was not sufficiently recognized until the 1980s when significant yield reduction observed in South Australia. Two main factors determining accumulation of high B level in soils are irrigation water (the most important contributor) and soils which inherently have high levels of B (Nable et al. 1997; Yau and Ryan 2008).

* Corresponding author; E-mail: mfbakhsh@ut.ac.ir 
Since ameliorating of B toxic soils is impractical in the most circumstances selecting or breeding of crop cultivars with high B toxicity tolerance is the only practical solution to increase yield in high B soils. Different works showed a wide range of B tolerance between and within plant species such as barely (Rehman et al. 2006), chickpea (Ardic et al. 2009), tomato (Güneş et al. 2009), canola (Öztürk et al. 2010), lentil (Hobson et al. 2006), maize (Hakki et al. 2007), Brassica rapa (Kaur et al. 2006), oilseed rape (Savić et al. 2013) and wheat (Karaman et al. 2012).

Karaman et al. (2012) reported that bread wheat genotypes are more B tolerant than durum wheat. Furlani et al. (2003) evaluated the response of four wheat genotypes to different B levels and concluded that IAC 287 and IAC 24 genotypes were more tolerant to B toxicity. Using 70 durum wheat genotypes in a greenhouse experiment to study genotype variation to B toxicity Torun et al. (2006) reported that dry matter production of all genotypes, except four, were markedly decreased by applied B. Kalayci et al. (1997) concluded that the most B tolerant wheat cultivars were of local origin. Breeding accessions of barely from Iran and Afghanistan were the most tolerant among seven WANA countries (Yau 2002).

Central and southern parts of Iran are typical arid and semi-arid areas with low rain fall. The soils of this area are characterized by low organic matter (OM) content, high soil $\mathrm{pH}$, high calcium carbonate content and coarse texture, which make them susceptible to induce B toxicity to plants, besides high levels of B in irrigation water and B toxicity symptoms reported from this area (Rajaie et al. 2009). In spite of wheat culture importance in central and southern parts of Iran no study on screening of B toxicity tolerance of Iranian wheat varieties has been reported. The current work was conducted to study differential responses of 10 Iranian wheat varieties to B toxicity in greenhouse conditions to select some cultivars having certain mechanisms controlling B toxicity for culture in soils containing toxic levels of B and find possible varieties for B toxicity tolerance breeding programs.

\section{Materials and Methods}

In order to compare B toxicity tolerance of some Iranian wheat varieties a greenhouse experiment was conducted with 10 wheat varieties, namely Verinac (V1), Hamoon (V2), Chamran (V3), Arg (V4), S7811 (V5), Pishtaz (V6), Parsi (V7), Arta (V8), Karkhe (V9) and Yavares (V10) (two last were durum wheat) and six B levels (0, 2.5, 5, 10, 20 and 40 $\mathrm{mg} \mathrm{B} \mathrm{kg}{ }^{-1}$ soil). Seeds were prepared from Seed Propagation Research Institute of Iran. The soil used in this study was from Iranian southern part $\left(28^{\circ} 32^{\prime} 48^{\prime \prime} \mathrm{N}\right.$ and $57^{\circ} 51^{\prime} 04^{\prime \prime} \mathrm{E}$ ), sandy loam in texture and 8\% clay content (Bouyoucos 1962). Some soil chemical properties (Sparks et al. 1996) were as: $\mathrm{EC}=3.5 \mathrm{dS} \mathrm{m}^{-1}, \mathrm{pH}$ (saturation paste $)=7.9$, saturation percentage $=27.9 \%$, organic carbon content $=0.62 \%$, cation exchange capacity $=8.12 \mathrm{cmol}_{\mathrm{c}} \mathrm{kg}^{-1}$, calcium carbonate equivalent $=4.90 \%$ and hot water soluble B $(\mathrm{HWSB})=0.9 \mathrm{mg} \mathrm{kg}^{-1}$.

Three $\mathrm{kg}$ of air dried soil replaced in plastic bags and $\mathrm{B}$ treatments as $\mathrm{H}_{3} \mathrm{BO}_{3}$ solution added to each one. In addition $35 \mathrm{mg} \mathrm{N} \mathrm{kg}^{-1}$ soil as urea, $50 \mathrm{mg} \mathrm{K} \mathrm{kg}^{-1}$ soil as $\mathrm{K}_{2} \mathrm{SO}_{4}$, 
$5 \mathrm{mg} \mathrm{Fe} \mathrm{kg}{ }^{-1}$ soil as FeEDHHA, $10 \mathrm{mg} \mathrm{Zn} \mathrm{kg}{ }^{-1}$ soil as $\mathrm{ZnSO}_{4}$ and $10 \mathrm{mg} \mathrm{Mn} \mathrm{kg}{ }^{-1}$ soil as $\mathrm{MnSO}_{4}$ were added to each pot uniformly. Soil in each bag reached to field capacity by adding distilled water and mixed thoroughly and translocated to poly ethylene pots. Two $35 \mathrm{mg} \mathrm{N} / \mathrm{kg}^{-1}$ soil as urea top dressed in three weeks intervals. Ten wheat seeds of each variety were planted in every pot and thinned to 5 uniform plants two week after planting. Day/night temperature was adjusted between $29 / 23{ }^{\circ} \mathrm{C}$ and $200 \mu \mathrm{mol} \mathrm{m} \mathrm{m}^{-2} \mathrm{~s}^{-1}$ irradiance with 12 hours photoperiod. Soil moisture controlled between $70-90 \%$ of field capacity (FC) by weighting pots and adding distilled water daily. Fifty-four days after sowing plants were cut from soil surface and shoot fresh weight determined, then washed thoroughly in tap and distilled water and oven dried in $65^{\circ} \mathrm{C}$ for 48 hours. Roots separated from soil and washed in tap and distilled water precisely to avoid any contamination and root dry weight and root B concentration determined. For B concentration measurement, $0.5 \mathrm{~g}$ of each of the shoot and root sample was dry-ashed in a muffle furnace at $500{ }^{\circ} \mathrm{C}$ for $6 \mathrm{~h}$ (Cottenie 1980). The ash was then dissolved in $0.1 \mathrm{~N} \mathrm{HCl}$ and $\mathrm{B}$ was determined colorimetrically $(420 \mathrm{~nm})$ using the azomethine-H method (Keren et al. 1996). Values were expressed as $\mathrm{mg} \mathrm{kg}^{-1} \mathrm{DW}$.

\section{Statistical analysis}

Recorded data were processed by the analysis of variance (ANOVA) in a factorial experiment on the basis of Completely Randomized Design (CRD) with 10 wheat varieties $\times$ six B levels $\left(0,2.5,5,10,20\right.$ and $40 \mathrm{mg} \mathrm{B} \mathrm{kg}^{-1}$ soil $)$ in 3 Replications and finally 180 pots. The data were analyzed by SAS 9.2 and Sigma Plot 12 computer soft wares. Treatment means were compared using LSD ( $t$-test). Slicing method was used for interaction analysis.

\section{Results}

The effect of B levels, variety and B levels $\times$ variety on shoot and root dry weight and shoot and root B concentration were significant. Increasing B levels up to $5 \mathrm{mg} \mathrm{kg}^{-1}$ did not affect shoot dry weight but higher B levels decreased shoot dry weight significantly (Table 1). In Chamran variety the second $\mathrm{B}$ level $\left(2.5 \mathrm{mg} \mathrm{kg}^{-1}\right)$ reduced shoot dry weight significantly that shows this variety is very sensitive to B toxicity. From the other side varieties like Arg, Karkhe, Arta, Pishtaz, S7811 and Hamoon showed significant dry weight reduction with increasing B level up to $20 \mathrm{mg} \mathrm{kg}^{-1}$, meaning that these varieties are relatively tolerant to B toxicity. Other varieties are between these two categories. Arg variety showed significantly higher shoot dry weight in different B levels than other varieties that shows B toxicity tolerance in this variety (Table 1). These results confirmed by shoot fresh weight results (data not showed). Root dry weight results were similar to shoot dry weight although it seems that B levels affect shoots more than roots. Increasing B levels up to $5 \mathrm{mg} \mathrm{kg}^{-1}$ did not affect root dry weight but higher B levels decreased root dry weight significantly. Arg variety produced significantly higher root dry weight than other varieties in different B levels (Table 1). 


\begin{tabular}{|c|c|c|c|c|c|c|c|c|c|c|c|c|c|c|c|c|c|c|c|c|c|c|c|}
\hline & 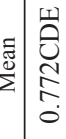 & $\begin{array}{l}\text { 핑 } \\
0 \\
0 \\
0 \\
0\end{array}$ & 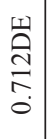 & $\underset{\Xi}{\stackrel{\leftrightarrows}{\leftrightarrows}}$ & 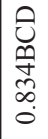 & 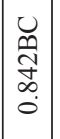 & 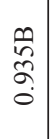 & $\begin{array}{l}\overrightarrow{2} \\
\stackrel{2}{0} \\
\stackrel{0}{\circ}\end{array}$ & $\begin{array}{l}0 \\
0 \\
n \\
\infty \\
0 \\
0\end{array}$ & 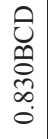 & & & $\begin{array}{l}\frac{1}{0} \\
\infty \\
\overrightarrow{0} \\
0\end{array}$ & 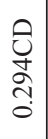 & 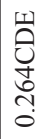 & 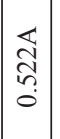 & 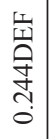 & 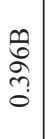 & 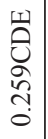 & 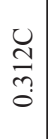 & 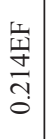 & 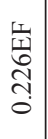 & \\
\hline & 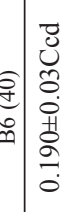 & 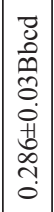 & 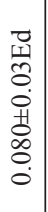 & 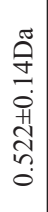 & 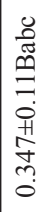 & $\begin{array}{c}0 \\
0 \\
0 \\
0 \\
0 \\
0 \\
01 \\
\tilde{O} \\
\tilde{0} \\
0\end{array}$ & 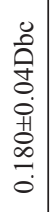 & 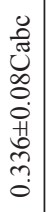 & 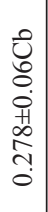 & 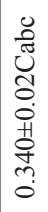 & $\begin{array}{l}\overrightarrow{8} \\
\delta \\
\\
0\end{array}$ & & 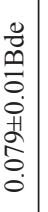 & 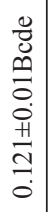 & $\begin{array}{l}0 \\
\stackrel{0}{0} \\
0 \\
\dot{0} \\
+1 \\
\stackrel{0}{0} \\
\dot{0} \\
\dot{0}\end{array}$ & 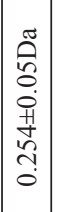 & 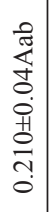 & $\begin{array}{l}0 \\
0 \\
0 \\
0 \\
0 \\
\dot{0} \\
2 \\
0 \\
0 \\
0\end{array}$ & $\begin{array}{l}0 \\
0 \\
0 \\
\dot{0} \\
\ddot{1} \\
\tilde{0} \\
\dot{0}\end{array}$ & 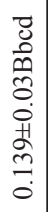 & 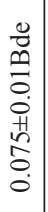 & 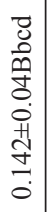 & $\begin{array}{l}\vec{D} \\
\stackrel{\infty}{\sim} \\
\stackrel{0}{0}\end{array}$ \\
\hline & 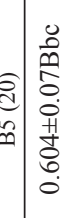 & 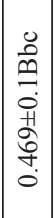 & $\begin{array}{c}0 \\
\hat{A} \\
0 \\
\dot{0} \\
+ \\
\stackrel{+}{f} \\
\dot{0}\end{array}$ & 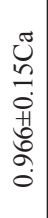 & 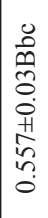 & $\left|\begin{array}{c}0 \\
0 \\
0 \\
0 \\
0 \\
0 \\
0 \\
0 \\
0 \\
0 \\
0 \\
0 \\
0\end{array}\right|$ & $\begin{array}{l}0 \\
0 \\
0 \\
0 \\
0 \\
\dot{0} \\
\stackrel{2}{1} \\
\tilde{c} \\
0\end{array}$ & 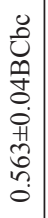 & 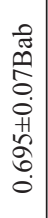 & 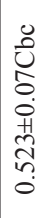 & 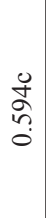 & & 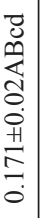 & 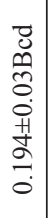 & 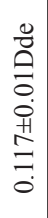 & $\left|\begin{array}{c}0 \\
0 \\
0 \\
0 \\
0 \\
0 \\
0 \\
0 \\
\infty \\
0 \\
0 \\
0\end{array}\right|$ & 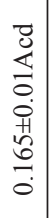 & 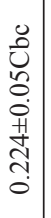 & 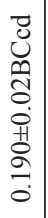 & 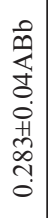 & 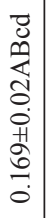 & $\begin{array}{c}\overrightarrow{0} \\
0 \\
0 \\
0 \\
0 \\
⿱ \\
\infty \\
\infty \\
\dot{0}\end{array}$ & $\begin{array}{l}\ddot{\infty} \\
\text { ஸे } \\
\text { ஸे }\end{array}$ \\
\hline 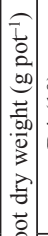 & 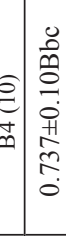 & 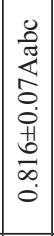 & 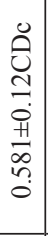 & 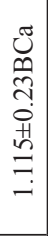 & 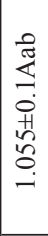 & 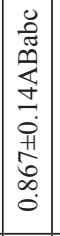 & 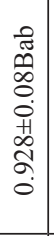 & 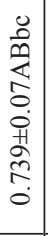 & 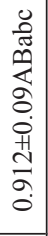 & 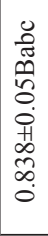 & 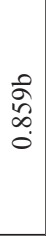 & 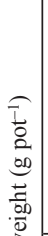 & 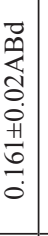 & 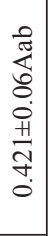 & 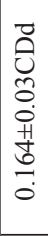 & $\begin{array}{c}\tilde{e} \\
0 \\
0 \\
0 \\
0 \\
n \\
0 \\
0 \\
0 \\
0\end{array}$ & 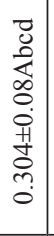 & 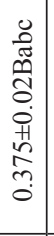 & 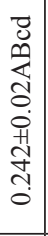 & 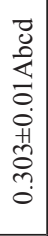 & 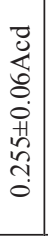 & 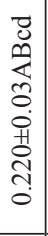 & 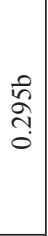 \\
\hline & 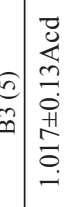 & 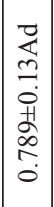 & 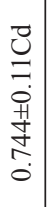 & 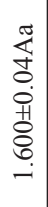 & 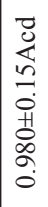 & 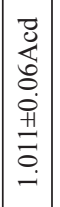 & 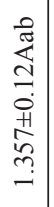 & 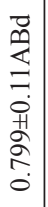 & 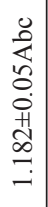 & 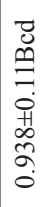 & 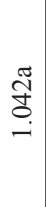 & 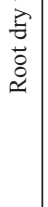 & 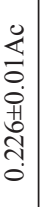 & 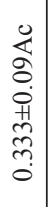 & 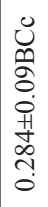 & 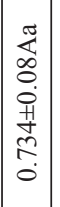 & 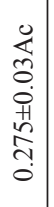 & 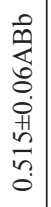 & 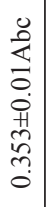 & 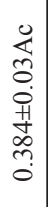 & 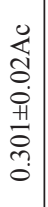 & 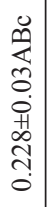 & $\begin{array}{l}\text { مू } \\
\text { ñ. } \\
0\end{array}$ \\
\hline & 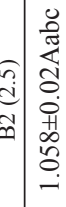 & 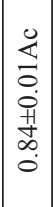 & 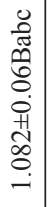 & $\begin{array}{l}\tilde{0} \\
\mathscr{0} \\
0 \\
0 \\
0 \\
+1 \\
0 \\
\stackrel{0}{-}\end{array}$ & 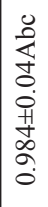 & 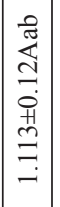 & 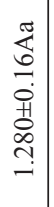 & 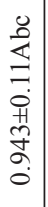 & 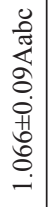 & 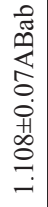 & 㞼 & & 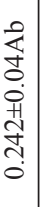 & 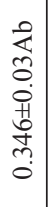 & 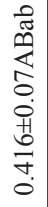 & $\mid \begin{array}{c}0 \\
0 \\
0 \\
0 \\
0 \\
0 \\
n \\
n \\
n \\
0\end{array}$ & 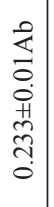 & $\begin{array}{c}\underset{⿱}{2} \\
\infty \\
0 \\
0 \\
0 \\
1 \\
\infty \\
n \\
n \\
0\end{array}$ & 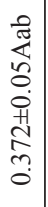 & 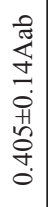 & 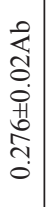 & 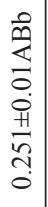 & 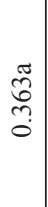 \\
\hline & êm & 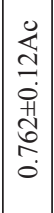 & 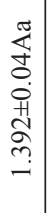 & 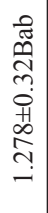 & 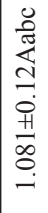 & 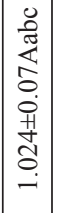 & 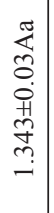 & 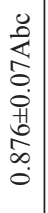 & 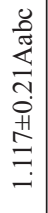 & 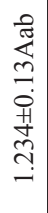 & $\begin{array}{l}\tilde{m} \\
\stackrel{\overbrace{}}{=}\end{array}$ & & 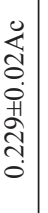 & 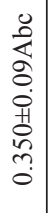 & 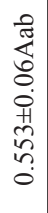 & 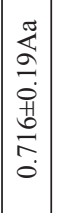 & 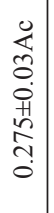 & 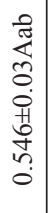 & 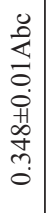 & 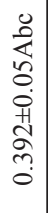 & 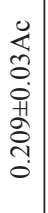 & 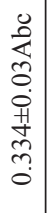 & ڤ్ \\
\hline & 莺 & 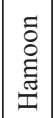 & 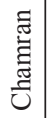 & $\stackrel{00}{\dot{Z}}$ & $\begin{array}{l}\bar{\nabla} \\
\infty \\
\infty\end{array}$ & 矛 & $\begin{array}{l}\vec{w} \\
\vec{E} \\
\vec{a}\end{array}$ & 焉 & 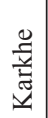 & 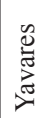 & $\Sigma$ & & 营 & 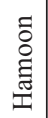 & 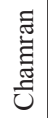 & 架 & $\begin{array}{l}\overrightarrow{\overrightarrow{0}} \\
\hat{\infty} \\
\infty\end{array}$ & 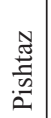 & $\begin{array}{l}\vec{w} \\
\overrightarrow{0} \\
\overrightarrow{0}\end{array}$ & 茕 & 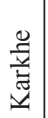 & 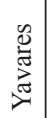 & $\stackrel{\Xi}{\Xi}$ \\
\hline
\end{tabular}




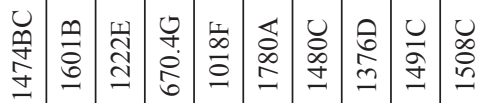

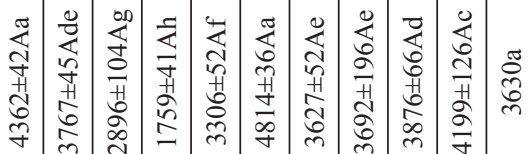

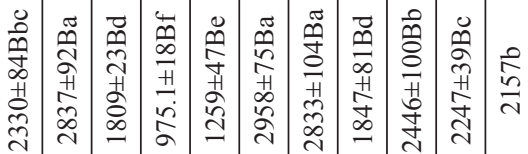

ลิ

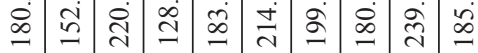

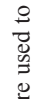

党

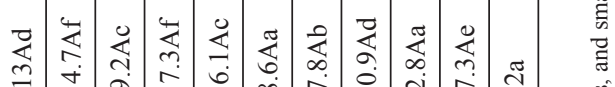

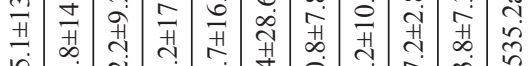

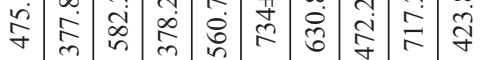

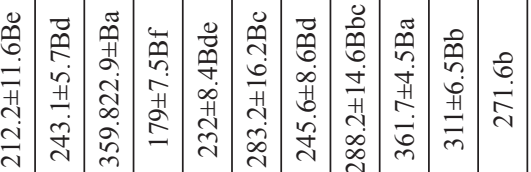

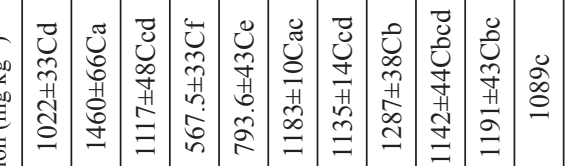

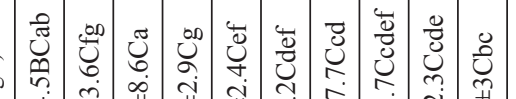

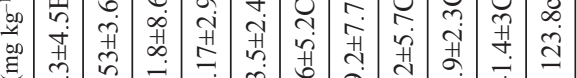

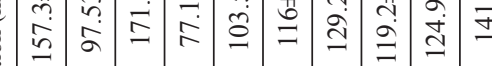
.

¿

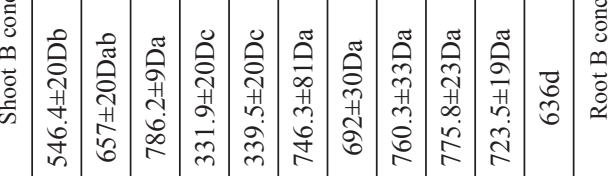

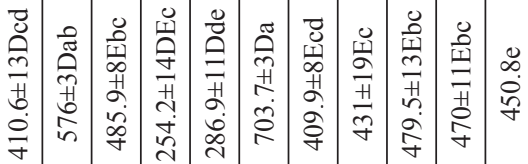

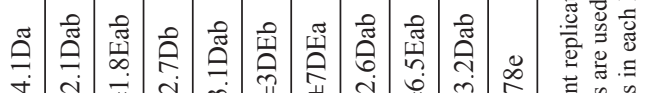

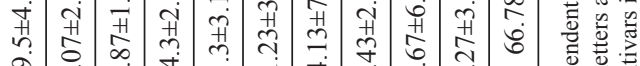

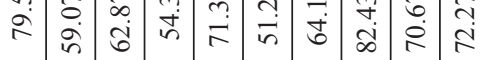

플

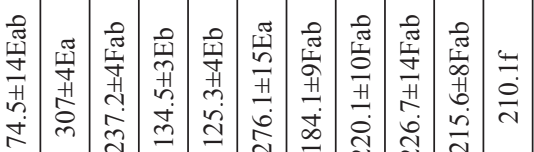

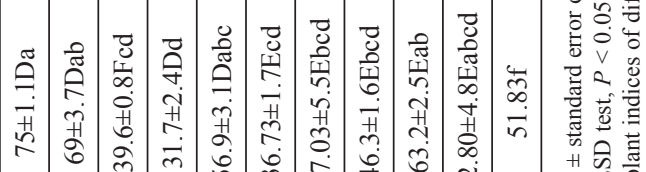

I

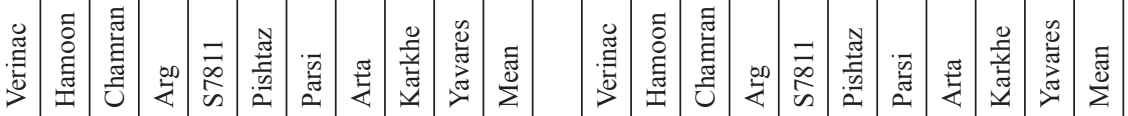




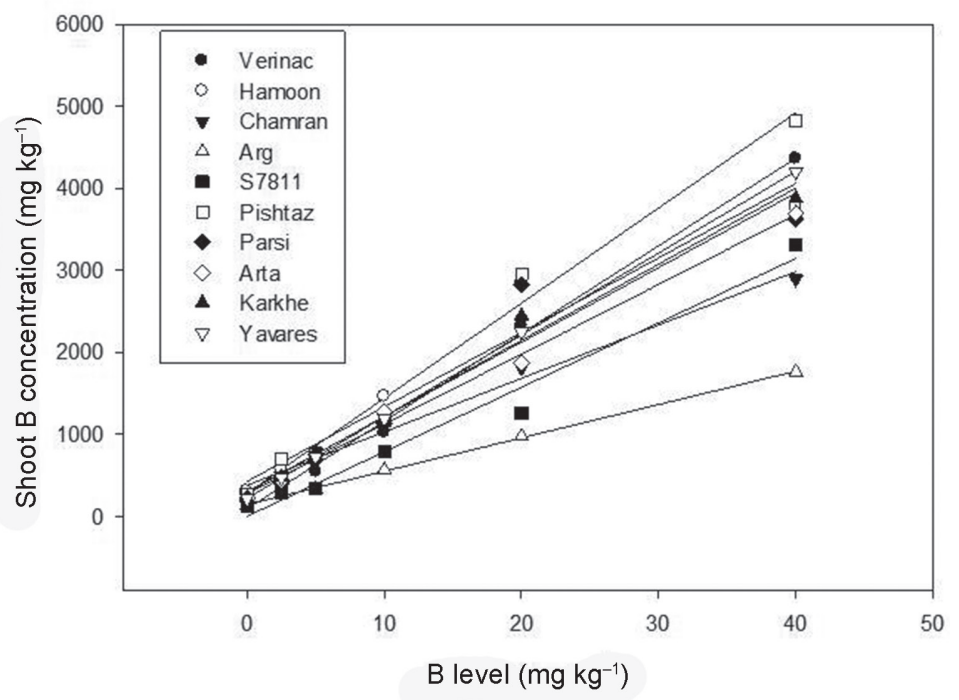

Figure 1. Effect of B levels on shoot B concentration in different wheat cultivars

Shoot and root B concentration increased significantly with increasing B levels (Table 1). Mean shoot and root B concentration of all genotypes correlated significantly to B application levels (Figs 1 and 2).

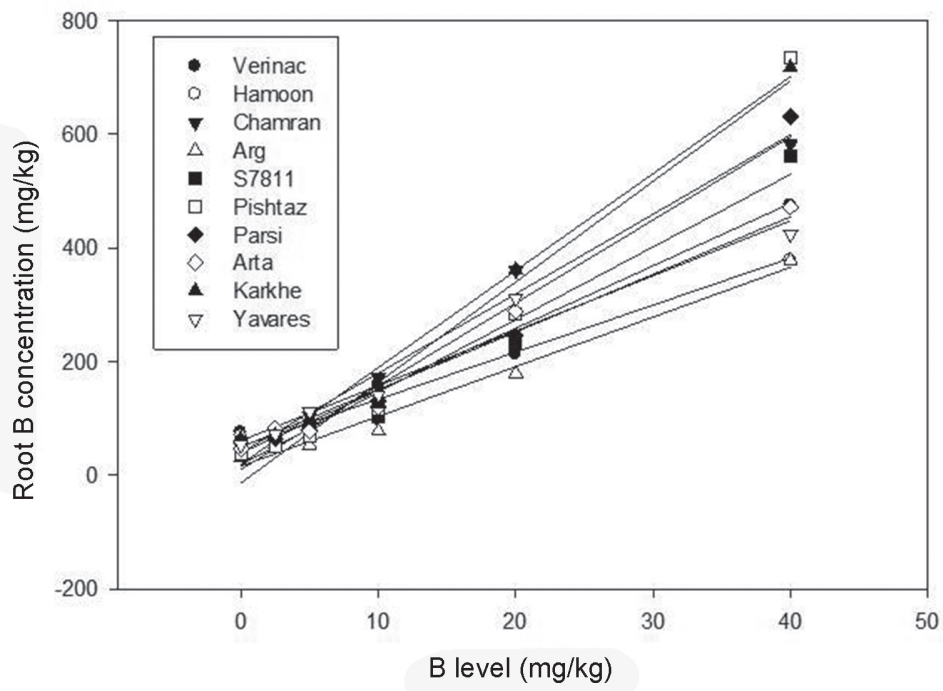

Figure 2. Effect of B levels on root B concentration in different wheat cultivars 
A great range of shoot and root B concentration observed in different wheat varieties. Mean shoot and root B concentration in different B levels ranged between 670.4-1780 $\mathrm{mg} \mathrm{kg}^{-1}$ and 128.8-239.1 $\mathrm{mg} \mathrm{kg}^{-1}$, respectively, for different wheat varieties (Table 1). There was a wider range for $\mathrm{B}$ concentration in shoot than root. The least shoot and root $\mathrm{B}$ concentration observed in Arg and the highest shoot and root B concentration were in Pishtaz and Karkhe respectively. Significant correlation $(r=0.93, p<0.01)$ observed between shoot and root $\mathrm{B}$ concentration and a negative significant correlation $(\mathrm{r}=0.85$, $p<0.01$ ) observed between shoot B concentration and shoot dry weight. Similarly significant negative correlation $(\mathrm{r}=0.66, p<0.01)$ observed between root $\mathrm{B}$ concentration and root dry weight.

Mean of shoot and root dry weight reduction percentage at two last B levels (20 and 40 $\mathrm{mg} \mathrm{kg}^{-1}$ ) ranged between $41.81-81.01 \%$ and $31.82-84.99 \%$, respectively (Table 2), which shows large variation in B toxicity tolerance among wheat varieties. Based on shoot dry weight data tolerance of different wheat varieties are as:

Arg $>$ Arta $>$ Pishtaz $>$ Hamoon $>$ Karkhe $>$ S7811 $>$ Verinac $>$ Yavares $>$ Parsi $>$ Chamran

Arg variety with minimum dry weight reduction for B highest levels had significantly higher shoot and root dry and fresh weight and significantly lower shoot and root B concentration than other varieties and can be considered as the most tolerant variety to $\mathrm{B}$ toxicity among experimental varieties. From the other side Chamran showed the highest dry weight reduction for highest $\mathrm{B}$ levels and can be considered as the most sensitive variety to B toxicity. Between two-tested durum wheat cultivar Karkhe is tolerant to B toxicity and can be cultivate in high B soils. A significant correlation observed between shoot $\mathrm{B}$ concentration and shoot dry weight reduction percentage $(\mathrm{r}=0.82, p<0.01)$.

Table 2. Reduction percentage of shoot and root dry weight in two highest B levels in different wheat varieties

\begin{tabular}{|l|c|c|c|c|c|c|}
\hline \multirow{2}{*}{} & \multicolumn{2}{|c}{ Shoot } & \multicolumn{2}{c|}{ Root } & \multicolumn{2}{c|}{ Mean } \\
\cline { 2 - 7 } & B5 (20) & B6 (40) & B5 (20) & B6 (40) & Shoot & Root \\
\hline Verinac & 41.02 & 81.45 & 25.18 & 65.36 & 61.23 & 45.27 \\
\hline Chamoon & 38.47 & 62.41 & 44.72 & 65.56 & 50.44 & 55.14 \\
\hline Arg & 67.91 & 94.11 & 78.84 & 91.14 & 81.01 & 84.99 \\
\hline S7811 & 24.44 & 59.18 & 45.74 & 64.56 & 41.81 & 55.15 \\
\hline Pishtaz & 48.47 & 67.87 & 39.88 & 23.76 & 58.17 & 31.82 \\
\hline Parsi & 41.83 & 57.13 & 59.06 & 70.90 & 49.48 & 64.98 \\
\hline Arta & 61.28 & 86.62 & 45.35 & 84.76 & 73.95 & 65.55 \\
\hline Karkhe & 35.76 & 61.68 & 27.74 & 64.51 & 48.72 & 46.12 \\
\hline Yavares & 37.87 & 75.11 & 19.01 & 64.22 & 56.44 & 41.62 \\
\hline
\end{tabular}




\section{Discussion}

In this study, the negative effect of high B levels on shoot and root growth varied among wheat varieties. Large variations in B toxicity tolerance among wheat varieties were seen for plant responses (Table 1). The second B level $\left(2.5 \mathrm{mg} \mathrm{kg}^{-1}\right)$ reduced shoot dry weight of Chamran variety significantly that shows this variety is very sensitive to B toxicity. There is increasing evidence showing that genotypes relatively sensitive to B toxicity are also relatively tolerant to B deficiency and vice versa (Yau 2002). On the other hand, this variety is B efficient and can be cultivated in low B soils and used as genetic material to breed varieties tolerant to B deficiency. Shoot and root dry weight of Arg variety in different B levels was significantly higher than other verities, and percent of shoot dry weight reduction in two highest $\mathrm{B}$ levels were minimum in this variety that shows this variety is the most tolerant to B toxicity among tested varieties. This variety could be cultivated in high B soils and could be recommended for breeding process for B toxicity tolerant genotypes. Metwally et al. (2012) concluded that high B levels reduced growth rate of B susceptible wheat Gemmeza 9 while increased tolerant sakha 93. They concluded adverse effect of high B levels on wheat growth seems to be cultivar dependent. Karaman et al. (2012) observed large genotypic variation among wheat genotypes to B toxicity. Variation of wheat varieties tolerance to B toxicity have been showed by others (Kalayci et al. 1997; Furlani et al. 2003; Torun et al. 2006).

Shoot and root B concentration increased significantly with increasing B levels and large variation in shoot and root B concentration observed in different wheat varieties. Significant increase in B concentration with increasing B levels and significant difference among wheat genotypes reported by Karaman et al. (2012).

Relative B toxicity tolerance of Arg, Karkhe, Arta, Pishtaz, S7811 and Hamoon varieties seems logical because they were evolved in dry areas where B toxicity is a problem which resulted to B toxicity tolerance of these varieties (Savić et al. 2013). Tolerant genotypes originated from regions where high B concentration has been reported in the soil and ground water (Nable et al. 1997). Yau (2002) reported that barley accessions from Iran and Afghanistan were the most tolerant among seven west Asia and north Africa countries.

There are some reports on less B toxicity tolerance of durum wheat compared to bread wheat (Kalayci et al. 1998; Karaman et al. 2012) but durum wheat Kharkhe showed relative B toxicity tolerance and can be cultivated in areas with high B levels.

Different mechanisms and/or genes are involved to induce B toxicity tolerance. Based on significant correlations between shoot B concentration and shoot dry weight reduction $(\mathrm{r}=0.82, p<0.01)$ and between shoot and root $\mathrm{B}$ concentration $(\mathrm{r}=0.92, p<0.01)$ it can be concluded that exclusion mechanism results to reduced concentration of $\mathrm{B}$ in $\mathrm{B}$ tolerant varieties. Kaur et al. (2006) in study of 19 Brassica rapa genotypes reported that tolerant genotypes used exclusion mechanism. Ardic et al. (2009) concluded that the ability of chick pea Gokce to reduce shoot B concentration by efflux of B from roots is the reason of improved growth of this genotype in B toxic condition. The primary mechanism for tolerance to B toxicity is the ability to maintain low concentrations of B in plant tissues. 
B toxicity tolerant varieties generally accumulate lower concentrations of B than sensitive ones. Active efflux is physiological reason for reduced B accumulation in tolerant varieties (Schnurbusch et al. 2010). Reid (2010) reported that wheat tolerance to B toxicity correlated with reduced tissue accumulation of $\mathrm{B}$. It can be concluded from the least shoot and root B concentration of Arg that B excludes from whole plant. However a closer look to shoot and root B concentration (Table 1) and reduction percentage of shoot dry weight with B levels (Table 2) shows in varieties like Pishtaz and Hamoon that exhibit remarkable $\mathrm{B}$ toxicity tolerance, $\mathrm{B}$ concentration is very high and this suggest a mechanism rather than exclusion. Torun et al. (2006) in a study of 70 durum wheat genotypes reported that some varieties showed a good relation between B concentration and decrease in dry weight but ultimately there was no significant relationship, and suggested that an internal mechanism is involved.

It seems that the mechanism is redistribution of B within leaf. Reid and Fitzpatrick (2009a and b) showed that redistribution of B from toxic place, symplast (cytoplasm) into less toxic place, apoplast (cell wall) reduces B toxicity. It seems that in some varieties like Arta, Karkhe and S7811 both mechanisms are involved to covey B toxicity tolerance. Existence of different $\mathrm{B}$ toxicity tolerance mechanisms give us chances to breed varieties with improved tolerance greater than that identified in this study.

Whether the best tolerant variety to B is also effective one for grain yield and how much $B$ is accumulated in the grain needs more prolonged experiments. To identify the nature of different tolerance to B some physiological and molecular approaches will be required.

In conclusion there was a great range in B toxicity tolerance among studied wheat varieties. Arg was the most tolerant one and Chamran showed the least tolerance. It seems that different mechanisms involved in B toxicity tolerance of wheat varieties. In some varieties (like Arg) exclusion of B from root, in some varieties (like Pishtaz and Hamoon) redistribution of $\mathrm{B}$ were the primary mechanisms, and in some varieties (like Arta, Karkhe, and S7811) both mechanisms are involved. The findings of this work are good base for future experiments in which one can concentrate on the two varieties Arg and Chamran as model plants suitable for experiments on different aspects of B tolerance.

\section{References}

Ardic, M., Sekmen, A.H., Tokur, S., Ozdemir, F., Turkan, I. 2009. Antioxidant responses of chickpea plants subjected to boron toxicity. Plant Biol. 11:328-338.

Avci, M., Akar, T. 2005. Severity and spatial distribution of boron toxicity in barley cultivated areas of Central Anatolia and transitional zones. Turkish J. of Agriculture and Forestry 29:377-382.

Bouyoucos, G.J. 1962. Hydrometer method improved for making particle size analyses of soils. Agron. J. 54:464-465.

Camacho-Cristóbal, J.J., Rexach, J., González-Fontes, A. 2008. Boron in plants: deficiency and toxicity. Journal of Integrative Plant Biol. 50:1247-1255.

Cottenie, A. 1980. Soil and plant testing as a basis of fertilizer recommendations. FAO Soils Bull. 38:70-73.

Furlani, A., Carvalho, C.P., de Freitas, J.G., Verdial, M.F. 2003. Wheat cultivar tolerance to boron deficiency and toxicity in nutrient solution. Scientia Agricola 60:359-370. 
Güneş, A., Alpaslan, M., Çikily, Y., Özcan, H. 2009. The effect of zinc on alleviation of boron toxicity in tomato plants (Lycopersicon esculentum L.). Turkish J. of Agriculture and Forestry 24:505-510.

Hakki, E., Atalay, E., Harmankaya, M., Babaoglu, M., Hamurcu, M., Gezgin, S., Kampus, K.T. 2007. Determination of suitable maize (Zea mays L.) genotypes to be cultivated in boron-rich In: Central Anatolian soil. Advances in Plant and Animal Boron Nutrition. Springer. Berlin, Germany. pp. 231-247.

Herrera-Rodríguez, M.B., González-Fontes, A., Rexach, J., Camacho-Cristóbal, J.J., Maldonado, J.M., Navarro-Gochicoa, M.T. 2010. Role of boron in vascular plants and response mechanisms to boron stresses. Plant Stress 4:115-122.

Hobson, K., Armstrong, R., Nicolas, M., Connor, D., Materne, M. 2006. Response of lentil (Lens culinaris) germplasm to high concentrations of soil boron. Euphytica 151:371-382.

Kalayci, M., Alkan, A., Cakmak, I., Bayramoğlu, O., Yilmaz, A., Aydin, M., Ozbek, V., Ekiz, H., Ozberisoy, F. 1997. Studies on differential response of wheat cultivars to boron toxicity. In: Wheat: Prospects for Global Improvement. Springer. Berlin, Germany. pp. 189-195.

Karaman, M.R., Zengin, M., Horuz, A. 2012. Assessment of resistance of wheat genotypes (T. aestivum and T. durum) to boron toxicity. In: Proceedings of World Academy of Science, Engineering and Technology: World Academy of Science, Engineering and Technology. Paris, France. pp. 809-812.

Kaur, S., Nicolas, M., Ford, R., Norton, R., Taylor, P. 2006. Selection of Brassica rapa genotypes for tolerance to boron toxicity. Plant and Soil 285:115-123.

Keren, R., Sparks, D., Page, A., Helmke, P., Loeppert, R., Soltanpour, P., Tabatabai, M., Johnston, C., Sumner, M. 1996. Boron. Methods of Soil Analysis. Part 3 - Chemical methods. pp. 603-626.

Metwally, A., El-Shazoly, R., Hamada, A.M. 2012. Effect of boron on growth criteria of some wheat cultivars. J. of Biology and Earth Sciences 2:B1-B9.

Nable, R.O., Bañuelos, G.S., Paull, J.G. 1997. Boron toxicity. Plant and Soil 193:181-198.

Öztürk, Ö., Soylu, S., Ada, R., Gezgin, S., Babaoglu, M. 2010. Studies on differential response of spring canola cultivars to boron toxicity. J. of Plant Nutrition 33:1141-1154.

Rajaie, M., Ejraie, A., Owliaie, H., Tavakoli, A. 2009. Effect of zinc and boron interaction on growth and mineral composition of lemon seedlings in a calcareous soil. International J. of Plant Prod. 3:39-50.

Rehman, S., Park, T.I., Kim, Y.J., Seo, Y.W., Yun, S.J. 2006. Inverse relationship between boron toxicity tolerance and boron contents of barley seed and root. J. of Plant Nutrition 29:1779-1789.

Reid, R. 2010. Can we really increase yields by making crop plants tolerant to boron toxicity? Plant Sci. 178:9-11.

Reid, R., Fitzpatrick, K. 2009a. Influence of leaf tolerance mechanisms and rain on boron toxicity in barley and wheat. Plant Physiol. 151:413-420.

Reid, R., Fitzpatrick, K. 2009b. Redistribution of boron in leaves reduces boron toxicity. Plant Signaling \& Behavior 4:1091-1093.

Savić, J., Marjanović-Jeromela, A., Glamočlija, Đ., Prodanović, S. 2013. Oilseed rape genotypes response to boron toxicity. Genetika 45:565-574.

Schnurbusch, T., Hayes, J., Sutton, T. 2010. Boron toxicity tolerance in wheat and barley: Australian perspectives. Breeding Science 60:297-304.

Sparks, D.L., Page, A., Helmke, P., Loeppert, R., Soltanpour, P., Tabatabai, M., Johnston, C., Sumner, M. 1996. Methods of Soil Analysis. Part 3. Chemichal methods. Soil Science Society of America. Book series No 5. Soil Science Society of America. Madison, WI 53711, USA. 1390 p.

Torun, A.A., Erdem, H., Çakmak, İ. 2006. Genotypic variation in tolerance to boron toxicity in 70 durum wheat genotypes. Turkish j. of Agriculture and Forestry 30:49-58.

Yau, S.K. 2002. Comparison of European with West Asian and North African winter barleys in tolerance to boron toxicity. Euphytica 123:307-314.

Yau, S.K., Ryan, J. 2008. Boron toxicity tolerance in crops: a viable alternative to soil amelioration. Crop Sci. 48:854-865. 